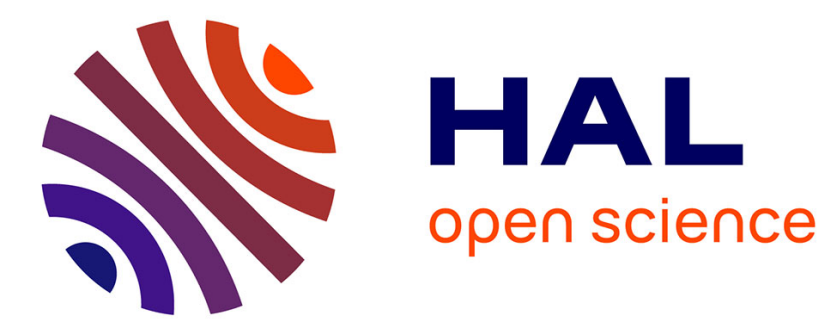

\title{
A Contractor Based on Convex Interval Taylor
}

\author{
Ignacio Araya, Gilles Trombettoni, Bertrand Neveu
}

\section{To cite this version:}

Ignacio Araya, Gilles Trombettoni, Bertrand Neveu. A Contractor Based on Convex Interval Taylor. [Research Report] RR-7887, INRIA. 2012, pp.23. hal-00673447

\section{HAL Id: hal-00673447 \\ https://hal.inria.fr/hal-00673447}

Submitted on 23 Feb 2012

HAL is a multi-disciplinary open access archive for the deposit and dissemination of scientific research documents, whether they are published or not. The documents may come from teaching and research institutions in France or abroad, or from public or private research centers.
L'archive ouverte pluridisciplinaire HAL, est destinée au dépôt et à la diffusion de documents scientifiques de niveau recherche, publiés ou non, émanant des établissements d'enseignement et de recherche français ou étrangers, des laboratoires publics ou privés. 


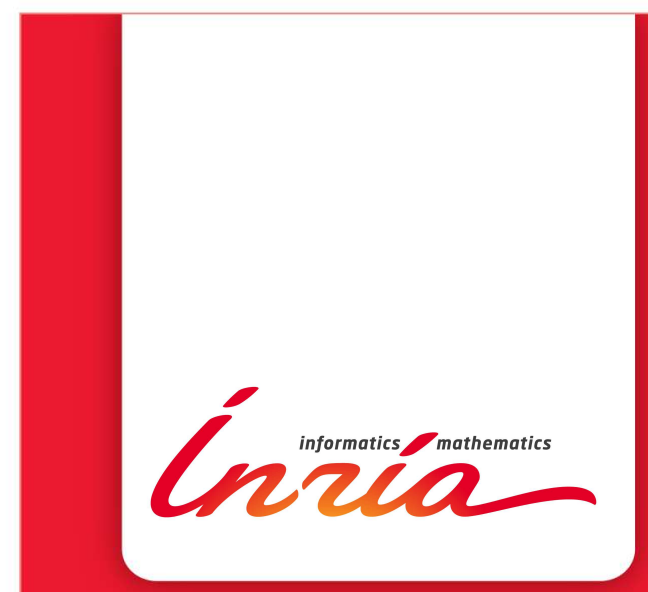

\section{A Contractor Based on Convex Interval Taylor}

Ignacio Araya, Gilles Trombettoni, Bertrand Neveu

\section{RESEARCH}

\section{REPORT}

$\mathrm{N}^{\circ} \mathbf{7 8 8 7}$

February 2012

Project-Team COPRIN 



\title{
inzéa
}

\section{A Contractor Based on Convex Interval Taylor}

\author{
Ignacio Araya*, Gilles Trombettoni甲 Bertrand Neved周 \\ Project-Team COPRIN
}

Research Report $n^{\circ} 7887$ - February $2012-23$ pages

\begin{abstract}
:
Interval Taylor has been proposed in the sixties by the interval analysis community for relaxing non-convex continuous constraint systems. However, it generally produces a non-convex relaxation of the solution set. A simple way to build a convex polyhedral relaxation is to select a corner of the studied domain/box as expansion point of the interval Taylor form, instead of the usual midpoint. The idea has been proposed by Neumaier to produce a sharp range of a single function and by Lin and Stadtherr to handle $n \times n$ (square) systems of equations.

This paper presents an interval Newton-like operator, called X-Newton, that iteratively calls this interval convexification based on an endpoint interval Taylor. This general-purpose contractor uses no preconditioning and can handle any system of equality and inequality constraints. It uses Hansen's variant to compute the interval Taylor form and uses two opposite corners of the domain for every constraint.

The X-Newton operator can be rapidly encoded, and produces good speedups in constrained global optimization and non-convex constraint satisfaction. First experiments compare X-Newton with affine arithmetic.
\end{abstract}

Key-words: intervals, Taylor, convex polyhedral relaxation, global optimization

\footnotetext{
* UTFSM, Chile. Email: iaraya@inf .utfsm.cl

$\dagger$ INRIA, I3S, Université Nice-Sophia, France. Email: Gilles. Trombet toni@inria.fr

* Imagine LIGM Université Paris-Est, France. Email: Bertrand. Neveu@enpc . fr
} 


\section{Un contracteur basé sur une forme de Taylor sur intervalles convexe}

Résumé : Une forme de Taylor sur intervalles a été proposée dans les années 1960 par la communauté de l'analyse par intervalles pour relaxer les systèmes de contraintes continues non convexes. Cependant, celle-ci produit généralement une relaxation non convexe de l'espace solution. Un moyen simple de produire une relaxation polyédrale convexe est de sélectionner un coin du domaine/boîte étudié comme point d'expansion de la forme de Taylor, en place du point milieu couramment utilisé. L'idée a été proposée par Neumaier pour calculer un intervalle étroit d'une fonction d'inclusion et par Lin et Stadtherr pour traiter des systèmes d'équations $n \times n$ (carrés).

Cet article présente un opérateur de Newton sur intervalles, appelé X-Newton, qui appelle itérativement cette convexification utilisant une forme de Taylor sur intervalles extrémale. Ce contracteur généraliste n'utilise pas de préconditionnement et peut traiter pratiquement n'importe quel système de contraintes d'égalité et d'inégalité. Il utilise la variante de E. R. Hansen pour calculer la forme de Taylor et utilise deux coins opposés du domaine pour chaque contrainte.

L'opérateur X-Newton peut se coder rapidement et apporte d'importantes accélérations en optimisation globale sous contraintes et en satisfaction de contraintes non convexes. De premières expérimentations comparent $\mathrm{X}$-Newton à l'arithmétique affine.

Mots-clés : intervalles, Taylor, relaxation polyédrale convexe, optimisation globale 


\section{Contents}

1 Motivation 3

\begin{tabular}{|lll}
\hline Background & 5
\end{tabular}

\begin{tabular}{|lll}
3 & Extremal interval Taylor form & $\mathbf{7}$
\end{tabular}

3.1 Corner selection for a tight convexification . . . . . . . . . . . . 7

3.2 Preliminary interval linearization . . . . . . . . . . . . . . . 10

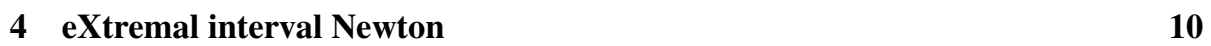

4.1 X-Newton iteration . . . . . . . . . . . . . . . . . 11

4.2 X-Newton . . . . . . . . . . . . . . . . . . 11

5 Experiments 12

5.1 Experiments in constrained global optimization . . . . . . . . . . . . 12

5.2 Experiments in constraint satisfaction . . . . . . . . . . . . . . 15

$\begin{array}{lll}6 & \text { Conclusion } & 18\end{array}$

A X-Newton and square systems of equations 21

A.1 Standard interval Newton . . . . . . . . . . . . . . . . . 21

A.2 I-Newton and X-Newton for square systems . . . . . . . . . . . . . 21

B Existence test for systems of inequality constraints 22

B.1 Adaptation to equality constraints . . . . . . . . . . . 23

\section{Motivation}

Interval B\&B algorithms are used to solve continous constraint systems and to handle constrained global optimization problems in a reliable way, i.e., they provide an optimal solution and its cost with a bounded error or a proof of infeasibility. The functions taken into account may be non-convex and can include many (piecewise) differentiable operators like arithmetic operators $(+,-, ., /)$, power, log, exp, sinus, etc.

Interval Newton is an operator often used by interval methods to contract/filter the search space [14]. The interval Newton operator uses an interval Taylor form to iteratively produce a linear system with interval coefficients. The main issue is that this system is not convex. Restricted to a single constraint, it forms a non-convex cone (a "butterfly"), as illustrated in Fig. 1-left. An n-dimensional constraint system is relaxed by an intersection of butterflies that is not convex either. (Examples can be found in [24, 15, 23].) Contracting optimally a box containing this non-convex relaxation has been proven to be NP-hard [16]. This explains why the interval analysis community has worked a lot on this problem for decades [14].

Only a few polynomial time solvable subclasses have been studied. The most interesting one has been first described by Oettli and Prager in the sixties [27] and occurs when the variables are all non-negative or non-positive. Unfortunately, when the Taylor expansion point is chosen strictly inside the domain (the midpoint typically), the studied box must be previously split into $2^{n}$ sub-problems/quadrants before falling in this interesting subclass [1, 4, 7]. Hansen and Bliek independently proposed a sophisticated and beautiful algorithm for avoiding explicitly handling the $2^{n}$ quadrants [13, 6]. 

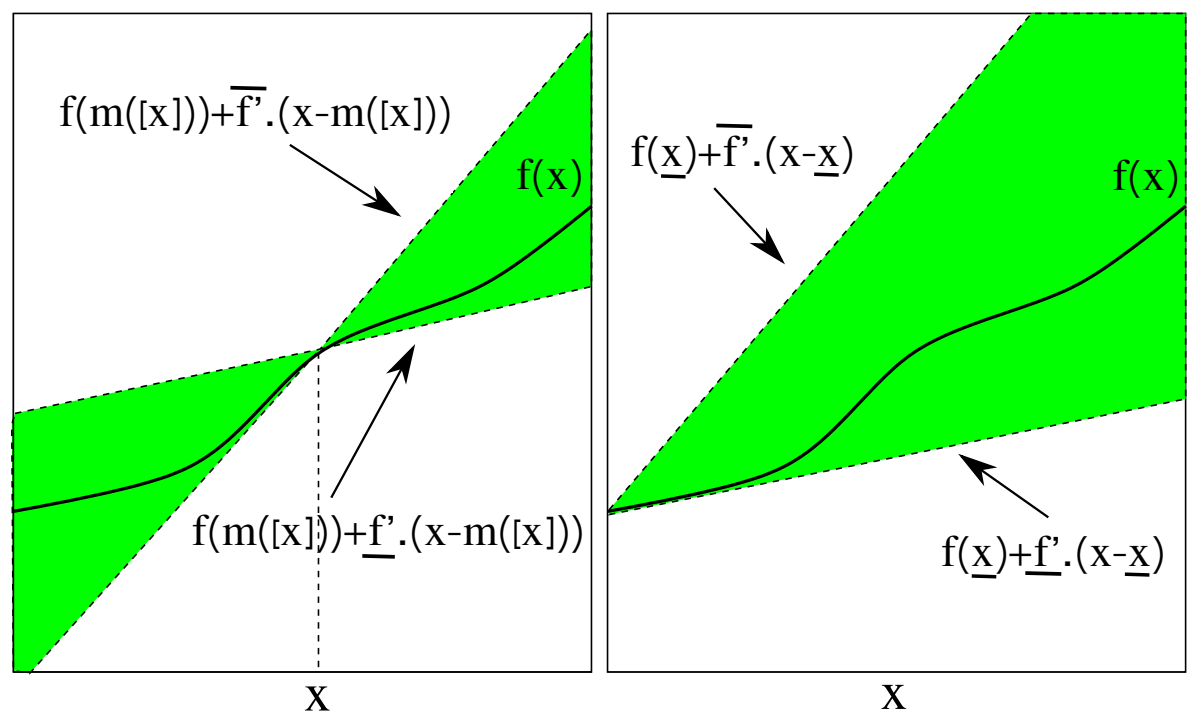

Figure 1: Relaxation of a function $f$ over the real numbers by a function $g: \mathbb{R} \rightarrow \mathbb{R}$ using an interval Taylor form (graph in gray). Left: Midpoint Taylor form, using a midpoint evaluation $f(m([x]))$, the maximum derivative $\overline{f^{\prime}}$ of $f$ inside the interval $[x]$ and the minimum derivative $f^{\prime}$. Right: Extremal Taylor form, using an endpoint evaluation $f(\underline{x}), \overline{f^{\prime}}$ and $\underline{f^{\prime}}$.

However, the method is restricted to $n \times n$ (square) systems of equations (no inequalities). 11 Also, the method requires the system be first preconditioned (i.e., the interval Jacobian matrix must be multiplied by the inverse matrix of the domain midpoint). The preconditioning has a cubic time complexity, implies an overestimate of the relaxation and requires non-singularity conditions often met only on small domains, at the bottom of the search tree.

In 2004, Lin \& Stadtherr [19] proposed to select a corner of the studied box, instead of the usual midpoint. Graphically, it produces a convex cone, as shown in Fig. 1-right. The main drawback of this extremal interval Taylor form is that it leads to a larger system relaxation surface. The main virtue is that the solution set belongs to a unique quadrant and is convex. It is a polytope that can be (box) hulled in polynomial-time by a linear programming (LP) solver: two calls to an LP solver compute the minimum and maximum values in this polytope for each of the $n$ variables (see Section 4). Upon this extremal interval Taylor, they have built an interval Newton restricted to square $n \times n$ systems of equations for which they had proposed in a previous work a specific preconditioning. They have presented a corner selection heuristic optimizing their preconditioning. The selected corner is common to all the constraints.

The idea of selecting a corner as Taylor expansion point is mentioned, in dimension 1, by A. Neumaier (see page 60 and Fig. 2.1 in [24]) for computing a range enclosure (see Def. 1) of a univariate function. Neumaier calls this the linear boundary value form. The idea has been exploited by Messine and Laganouelle for lower bounding the objective function in a Branch \& Bound algorithm for unconstrained global optimization [21].

${ }^{1}$ It could be applied to under-constrained systems of equations by using techniques described in [11] 
McAllester et al. also mention this idea in [20] (end of page 2) for finding cuts of the box in constraint systems. At page 211 of Neumaier's book [24], the step (4) of the presented pseudo-code also uses an endpoint interval Taylor form for contracting a system of equations ${ }^{2}$

\section{Contributions}

We present in this paper a new contractor, called X-Newton (for eXtremal interval Newton), that iteratively achieves an interval Taylor form on a corner of the studied domain. X-Newton does not require the system be preconditioned and can thus reduce the domains higher in the search tree. It can treat well-constrained systems as well as under-constrained ones (with fewer equations than variables and with inequalities), as encountered in constrained global optimization. The only limit is that the domain must be bounded, although the considered intervals, i.e., the initial search space, can be very large.

This paper experimentally shows that such a contractor is crucial in constrained global optimization and is also useful in continuous constraint satisfaction where it makes the whole solving strategy more robust.

After the background introduced in the next section, we show in Section 3 that the choice of the best expansion corner for any constraint is an NP-hard problem and propose a simple selection policy choosing two opposite corners of the box. Tighter interval partial derivatives are also produced by Hansen's recursive variant of interval Taylor. Section 4 details the extremal interval Newton operator that iteratively computes a convex interval Taylor form. Section 5 highlights the benefits of X-Newton in satisfaction and constrained global optimization problems.

This work provides an alternative to the two existing reliable (interval) convexification methods used in global optimization. The Quad [18, 17] method is an interval reformulation-linearization technique that produces a convex polyhedral approximation of the quadratic terms in the constraints. Affine arithmetic produces a polytope by replacing in the constraint expressions every basic operator by specific affine forms [10, 33, 3]. It has been recently implemented in an efficient interval B\&B [26]. Experiments provide a first comparison between this affine arithmetic and the cornerbased interval Taylor.

\section{Background}

Intervals allow reliable computations on computers by managing floating-point bounds and outward rounding.

\section{Intervals}

An interval $\left[x_{i}\right]=\left[x_{i}, \overline{x_{i}}\right]$ defines the set of reals $x_{i}$ s.t. $x_{i} \leq x_{i} \leq \overline{x_{i}}$, where $x_{i}$ and $\overline{x_{i}}$ are floating-point numbers. $\mathbb{I R}$ denotes the set of all intervals. The size or width of $\left[x_{i}\right]$ is $w\left(\left[x_{i}\right]\right)=\overline{x_{i}}-x_{i}$. A box $[x]$ is the Cartesian product of intervals $\left[x_{1}\right] \times \ldots \times\left[x_{i}\right] \times \ldots \times\left[x_{n}\right]$.

\footnotetext{
${ }^{2}$ The aim is not to produce a convex polyhedral relaxation (which is not mentioned), but to use as expansion point the farthest point in the domain from a current point followed by the algorithm. The contraction is not obtained by calls to an LP solver but by the general purpose Gauss-Seidel without taking advantage of the convexity.
} 
Its width is defined by $\max _{i} w\left(\left[x_{i}\right]\right) . m([x])$ denotes the middle of $[x]$. The hull of a subset $S$ of $\mathbb{R}^{n}$ is the smallest $\mathrm{n}$-dimensional box enclosing $S$.

Interval arithmetic [22] has been defined to extend to $\mathbb{I} \mathbb{R}$ elementary functions over $\mathbb{R}$. For instance, the interval sum is defined by $\left[x_{1}\right]+\left[x_{2}\right]=\left[\underline{x_{1}}+\underline{x_{2}}, \overline{x_{1}}+\overline{x_{2}}\right]$. When a function $f$ is a composition of elementary functions, an extension of $f$ to intervals must be defined to ensure a conservative image computation.

Definition 1 (Extension of a function to $\mathbb{I}$; inclusion function; range enclosure) Consider a function $f: \mathbb{R}^{n} \rightarrow \mathbb{R}$.

$[f]: \mathbb{\mathbb { R } ^ { n }} \rightarrow \mathbb{R}$ is said to be an extension of $f$ to intervals iff:

$$
\begin{array}{ll}
\forall[x] \in \mathbb{R}^{n} & {[f]([x]) \supseteq\{f(x), x \in[x]\}} \\
\forall x \in \mathbb{R}^{n} & f(x)=[f](x)
\end{array}
$$

The natural extension $[f]_{N}$ of a real function $f$ corresponds to the mapping of $f$ to intervals using interval arithmetic. The outer and inner interval linearizations proposed in this paper are related to the first-order interval Taylor extension [22], defined as follows:

$$
[f]_{T}([x])=f(\dot{x})+\sum_{i}\left[a_{i}\right] \cdot\left(\left[x_{i}\right]-\dot{x}_{i}\right)
$$

where $\dot{x}$ denotes any point in $[x]$, e.g., $m([x])$, and $\left[a_{i}\right]$ denotes $\left[\frac{\partial f}{\partial x_{i}}\right]_{N}([x])$.

Equivalently, we have: $\forall x \in[x], \underline{[f]_{T}([x])} \leq f(x) \leq \overline{[f]_{T}([x])}$.

Example. Consider $f\left(x_{1}, x_{2}\right)=3 x_{1}^{2}+x_{2}^{2}+x_{1} x_{2}$ in the box $[x]=[-1,3] \times[-1,5]$. The natural evaluation provides: $[f]_{N}\left(\left[x_{1}\right],\left[x_{2}\right]\right)=3[-1,3]^{2}+[-1,5]^{2}+[-1,3][-1,5]=$ $[0,27]+[0,25]+[-5,15]=[-5,67]$. The partial derivatives are: $\frac{\partial f}{\partial x_{1}}\left(x_{1}, x_{2}\right)=6 x_{1}+x_{2}$, $\left[\frac{\partial f}{\partial x_{1}}\right]_{N}([-1,3],[-1,5])=[-7,23], \frac{\partial f}{\partial x_{2}}\left(x_{1}, x_{2}\right)=x_{1}+2 x_{2},\left[\frac{\partial f}{\partial x_{2}}\right]_{N}\left(\left[x_{1}\right],\left[x_{2}\right]\right)=[-3,13]$. The interval Taylor evaluation with $\dot{x}=m([x])=(1,2)$ yields: $[f]_{T}\left(\left[x_{1}\right],\left[x_{2}\right]\right)=9+$ $[-7,23][-2,2]+[-3,13][-3,3]=[-76,94]$.

\section{A simple convexification based on interval Taylor}

Consider a function $f: \mathbb{R}^{n} \rightarrow \mathbb{R}$ defined on a domain $[x]$, and the inequality constraint $f(x) \leq 0$. For any variable $x_{i} \in x$, let us denote $\left[a_{i}\right]$ the interval partial derivative $\left[\frac{\partial f}{\partial x_{i}}\right]_{N}([x])$. The first idea is to lower tighten $f(x)$ with one of the following interval linear forms that hold for all $x$ in $[x]$.

$$
\begin{aligned}
f(\underline{x})+\underline{a_{1}} y_{1}^{l}+\ldots+\underline{a_{n}} y_{n}^{l} \leq f(x) \\
f(\bar{x})+\overline{a_{1}} y_{1}^{r}+\ldots+\overline{a_{n}} y_{n}^{r} \leq f(x)
\end{aligned}
$$

where: $y_{i}^{l}=x_{i}-\underline{x_{i}}$ and $y_{i}^{r}=x_{i}-\overline{x_{i}}$.

A corner of the box is chosen: $\underline{x}$ in form (1) or $\bar{x}$ in form (2). When applied to a set of inequality and equality ${ }^{3}$ constraints, we obtain a polytope enclosing the solution set.

The correctness of relation (11) - see for instance [31, 19] - lies on the simple fact that any variable $y_{i}^{l}$ is non-negative since its domain is $\left[0, d_{i}\right]$, with $d_{i}=w\left(\left[y_{i}^{l}\right]\right)=$ $w\left(\left[x_{i}\right]\right)=\overline{x_{i}}-\underline{x_{i}}$. Therefore, minimizing each term $\left[a_{i}\right] y_{i}^{l}$ for any point $y_{i}^{l} \in\left[0, d_{i}\right]$ is

\footnotetext{
${ }^{3}$ An equation $f(x)=0$ can be viewed as two inequality constraints: $0 \leq f(x) \leq 0$.
} 
obtained with $a_{i}$. Symmetrically, relation 22 is correct since $y_{i}^{r} \in\left[-d_{i}, 0\right] \leq 0$, and the minimal value of a term is obtained with $\overline{a_{i}}$.

Note that, even though the polytope computation is safe, the floating-point roundoff errors made by the LP solver could render the hull of the polytope unsafe. A cheap post-processing proposed in [25], using interval arithmetic, is added to guarantee that no solution is lost by the Simplex algorithm.

\section{Extremal interval Taylor form}

\subsection{Corner selection for a tight convexification}

Relations (1) and 22 consider two specific corners of the box $[x]$. We can remark that every other corner of $[x]$ is also suitable. In other terms, for every variable $x_{i}$, we can indifferently select one of both bounds of $\left[x_{i}\right]$ and combine them in a combinatorial way: either $x_{i}$ in a term $a_{i}\left(x_{i}-x_{i}\right)$, like in relation $(1)$, or $\overline{x_{i}}$ in a term $\overline{a_{i}}\left(x_{i}-\overline{x_{i}}\right)$, like in relation $[\overline{2]}$.

A natural question then arises: Which corner $x^{c}$ of $[x]$ among the $2^{n}$-set $X^{c}$ ones produces the tightest convexification? If we consider an inequality $f(x) \leq 0$, we want to compute a hyperplane $f^{l}(x)$ that approximates the function, i.e., for all $x$ in $[x]$ we want: $f^{l}(x) \leq f(x) \leq 0$.

Following the standard policy of linearization methods, for every inequality constraint, we want to select a corner $x^{c}$ whose corresponding hyperplane is the closest to the non-convex solution set, i.e., adds the smallest volume. This is exactly what represents Expression (3) that maximizes the Taylor form for all the points $x=\left\{x_{1}, \ldots, x_{n}\right\} \in$ $[x]$ and adds their different contributions: one wants to select a corner $x^{c}$ from the set of corners $X^{c}$ such that:

$$
\max _{x^{c} \in X^{c}} \int_{x_{1}=\underline{x_{1}}}^{\overline{x_{1}}} \ldots \int_{x_{n}=\underline{x_{n}}}^{\overline{x_{n}}}\left(f\left(x^{c}\right)+\sum_{i} z_{i}\right) d x_{n} \ldots d x_{1}
$$

where: $z_{i}=\overline{a_{i}}\left(x_{i}-\overline{x_{i}}\right)$ iff $x_{i}^{c}=\overline{x_{i}}$, and $z_{i}=\underline{a_{i}}\left(x_{i}-\underline{x_{i}}\right)$ iff $x_{i}^{c}=\underline{x_{i}}$.

Since:

- $f\left(x^{c}\right)$ is independent from the $x_{i}$ values,

- any point $z_{i}$ depends on $x_{i}$ but does not depend on $x_{j}$ (with $j \neq i$ ),

- $\int_{x_{i}=\underline{x_{i}}}^{\overline{x_{i}}} \underline{a_{i}}\left(x_{i}-\underline{x_{i}}\right) d x_{i}=\underline{a_{i}} \int_{y_{i}=0}^{d_{i}} y_{i} d y_{i}=\underline{a_{i}} 0.5 d_{i}^{2}$,

- $\int_{x_{i}=x_{i}}^{\overline{x_{i}}} \overline{a_{i}}\left(x_{i}-\overline{x_{i}}\right) d x_{i}=\overline{a_{i}} \int_{-d_{i}}^{0} y_{i} d y_{i}=-0.5 \overline{a_{i}} d_{i}^{2}$,

Expression (3) is equal to:

$$
\max _{x^{c} \in X^{c}} \prod_{i} d_{i} f\left(x^{c}\right)+\prod_{i} d_{i} \sum_{i} 0.5 a_{i}^{c} d_{i}
$$

where $d_{i}=w\left(\left[x_{i}\right]\right)$ and $a_{i}^{c}=\underline{a_{i}}$ or $a_{i}^{c}=-\overline{a_{i}}$.

We simplify by the positive factor $\prod_{i} d_{i}$ and obtain:

$$
\max _{x^{c} \in X^{c}} f\left(x^{c}\right)+0.5 \sum_{i} a_{i}^{c} d_{i}
$$




\section{Tightest corner convexification is NP-hard}

Unfortunately, we can prove that this maximization problem (4) is NP-hard. The following lemma underlines that the difficult part is to maximize $f\left(x^{c}\right)$.

Lemma 1 Consider a polynomial function $f: \mathbb{R}^{n} \rightarrow \mathbb{R}$, with rational coefficients, and defined on a domain $[x]=[0,1]^{n}$. Let $X^{c}$ be the $2^{n}$-set of corners, i.e., in which every element is a bound 0 or 1 . Then,

$\max _{x^{c} \in X^{c}}-f\left(x^{c}\right)$ (or $\min _{x^{c} \in X^{c}} f\left(x^{c}\right)$ ) is an NP-hard problem.

The result is probably well-known but we are interested here in the reduction.

Proof. We prove that the (minimization) problem of finding a corner $x^{c} \in X^{c}$ such that $f\left(x^{c}\right) \leq B$ (where $B$ is a rational bound ${ }^{4}$ is as hard as the well-known NP-complete 3SAT problem. The polynomial reduction from a 3SAT instance $I$ to a corner selection instance $I^{\prime}$ is the following:

- An instance $I$ of 3SAT is given by a set of $n$ boolean variables $\left\{x_{1}, \ldots, x_{i}, \ldots, x_{n}\right\}$ and a BNF boolean formula, i.e., a conjunction of clauses $C_{I}=\bigwedge_{j}\left(l_{1}^{j} \vee l_{2}^{j} \vee l_{3}^{j}\right)$, where $l_{k}^{j}$ denotes a positive literal $x_{i}$ or a negative literal $\neg x_{i}$.

- For every boolean variable $x_{i}$ in $I$, a rational variable $x_{i}^{\prime}$ is generated in $I^{\prime}$ with domain $[0,1]$.

- A boolean formula $C_{I}$ is reduced to a polynomial inequality made of a sum of products: $\sum_{j}\left(x_{1}^{\prime j} x_{2}^{\prime j} x_{3}^{\prime j}\right) \leq 0$. For every clause $c_{j}=\left(l_{1}^{j} \vee l_{2}^{j} \vee l_{3}^{j}\right)$ of $C_{I}$, we generate a term $\left(x_{1}^{\prime j} x_{2}^{\prime j} x_{3}^{\prime j}\right)$ where:

$$
\begin{aligned}
& -x_{k}^{\prime j}=1-x_{i}^{\prime} \text { if } l_{k}^{j}=x_{i} \text { is a positive literal in } c_{j}, \\
& -x_{k}^{\prime j}=x_{i}^{\prime} \text { if } l_{k}^{j}=\neg x_{i} \text { is a negative literal in } c_{j} .
\end{aligned}
$$

- Note that we have chosen the bound $B=0$.

It is straightforward (a) to check that this tranformation is polynomial, (b) to check in polynomial-time the existence of a solution of $I^{\prime}$ and (c) that a solution of an instance $I$ is equivalent to a solution of an instance $I^{\prime}$. Indeed:

- A boolean variable $x_{i}$ is true (resp. false) iff $x_{i}^{\prime}=1$ (resp. $x_{i}^{\prime}=0$ ).

- A literal in a clause $c_{j}$ is true iff the corresponding term $x_{1}^{\prime j} x_{2}^{\prime j} x_{3}^{\prime j}=0$.

- The conjunction $C_{I}$ is satisfiable iff all terms in $I^{\prime}$ are null $\left(f\left(x^{c}\right) \leq 0\right)$.

On the other hand, it is easy to maximize the other term $0.5 \sum_{i} a_{i}^{c} d_{i}$ in Expression 4 by selecting the maximum value among $\underline{a_{i}}$ and $-\overline{a_{i}}$ in every term.

The difficulty is thus to determine the computational complexity of the problem (4) that combines $f\left(x^{c}\right)$ (NP-hard) and $0.5 \sum_{i} a_{i}^{c} d_{i}$ (in $P$ ). In order to prove the NP-hardness of the problem (4), our first (failed) idea was to achieve a polynomial tranformation in which the derivative part $0.5 \sum_{i} a_{i}^{c} d_{i}$ would be always negligible over its counterpart in $f\left(x^{c}\right)$. Instead, we propose a polynomial reduction in which the derivative part is constant, i.e., $\forall_{i} \underline{a_{i}}=-\overline{a_{i}}$. Thus:

\footnotetext{
${ }^{4}$ We "restrict" the class to polynomial functions, otherwise the corresponding decision problem would not belong to NP. Indeed, verifying the satisfaction of a constraint with, e.g., trigonometric operators cannot be achieved in polynomial-time due to considerations related to floating-point calculation.
} 
Proposition 1 (Corner selection is NP-hard)

Consider a polynomial ${ }^{5} f: \mathbb{R}^{n} \rightarrow \mathbb{R}$, with rational coefficients, and defined on a domain $[x]=[0,1]^{n}$. Let $X^{c}$ be the $2^{n}$-set of corners, i.e., in which every component is a bound 0 or 1 . Then,

$$
\begin{aligned}
& \max _{x^{c} \in X^{c}}-\left(f\left(x^{c}\right)+0.5 \sum_{i} a_{i}^{c} d_{i}\right) \\
& \left(\text { or } \min _{x^{c} \in X^{c}} f\left(x^{c}\right)+0.5 \sum_{i} a_{i}^{c} d_{i}\right)
\end{aligned}
$$

is an NP-hard problem.

Proof. The polynomial reduction have similarities with the reduction shown in Lemma1. The main difference is that we consider a subclass of 3SAT, called here BALANCED3SAT. In an instance of BALANCED-3SAT, each boolean variable $x_{i}$ occurs $n_{i}$ times in a negative literal and $n_{i}$ times in a positive literal. We know that BALANCED-3SAT is NP-complete thanks to the dichotomy theorem by Thomas J. Schaefer who identified the only 6 subclasses of SAT that are in $P[29]$. BALANCED-3SAT does not belong to none of these 6 subclasses 6

Considering $f\left(x^{c}\right)+0.5 \sum_{i} a_{i}^{c} d_{i} \leq B$, a second difference with Lemma 1 is the chosen bound $B$. We choose $B=0.5 \sum_{i} d_{i}\left(-n_{i}\right)=-0.5 \sum_{i} n_{i}$ (recall that $\forall i, d_{i}=1$ ).

It is less trivial to check that a solution of an instance $I$ of BALANCED-3SAT is equivalent to a solution of an instance $I^{\prime}$ of $f\left(x^{c}\right)+0.5 \sum_{i} a_{i}^{c} d_{i} \leq-0.5 \sum_{i} n_{i}$. Each term $x_{1}^{\prime j} x_{2}^{\prime j} x_{3}^{\prime j}$ of $I^{\prime}$ implies a partial derivative $\frac{\partial f}{\partial x_{i}^{\prime}}([x])$ equal to 0 if $x_{i}^{\prime}$ does not appear in the term, equal to $[-1,0]$ if $x_{i}$ appears as a positive literal in $I$ (i.e., $x_{k}^{\prime j}=\left(1-x_{i}^{\prime}\right)$ and $[-1,0]=-1[0,1][0,1]$ ), and equal to $[0,1]$ if $x_{i}$ appears as a negative literal (i.e., $x_{k}^{\prime j}=x_{i}^{\prime}$ and $\left.[0,1]=1[0,1][0,1]\right)$. Thus, by adding all these intervals in the different terms, we obtain $\left[a_{i}\right]=\left[-n_{i}, n_{i}\right]$ and thus $\forall_{i} \underline{a_{i}}=-\overline{a_{i}} \square$

\section{Using two opposite corners}

Even more annoying is that experiments presented in Section 5 suggest that the criterion (4) is not relevant in practice. Indeed, even if the best corner was chosen (by an oracle), the gain in box contraction brought by this strategy w.r.t. a random choice of corner would be not significant. This renders pointless the search for an efficient and fast corner selection heuristic.

This study suggests that this criterion is not relevant and leads to explore another criterion. We should notice that when a hyperplane built by endpoint interval Taylor removes some inconsistent parts from the box, the inconsistent subspace more often includes the selected corner $x_{c}$ because the approximation at this point is exact. However, the corresponding criterion includes terms mixing variables coming from all the dimensions simultaneously, and makes difficult the design of an efficient corner selection heuristic. This qualitative analysis nevertheless provides us rationale to adopt the following policy.

To obtain a better contraction, it is also possible to produce several, i.e., $c$, linear expressions lower tightening a given constraint $f(x) \leq 0$. Applied to the whole system

\footnotetext{
${ }^{5}$ We cannot prove anything on more complicated, e.g., transcendental, functions that make the problem undecidable.

${ }^{6} \mathrm{~A}$ straightforward reduction from 3SAT to BALANCED-3SAT could also be followed: add to the 3SAT instance $d$ "dummy" clauses, one for each "missing" literal; for one such literal, e.g., $\neg x_{i}$, the corresponding clause is $\neg x_{i} \vee b_{j} \vee \neg b_{j-1}$; the $b_{j}$ variables $(j \in\{1 \ldots d\})$ are dummy additional boolean variables (appearing $d$ times as a negative literal and $d$ times as a positive literal in round-robin...).
} 
with $m$ inequalities, the obtained polytope corresponds to the intersection of these $\mathrm{cm}$ half-spaces. Experiments (see Section 5.2) suggest that generating two hyperplanes (using two corners) yields a good ratio between contraction (gain) and number of hyperplanes (cost). Also, choosing opposite corners tends to minimize the redundancy between hyperplanes since the hyperplanes remove from the box preferably the search subspaces around the selected corners.

Note that, for managing several corners simultaneously, an expanded form must be adopted to put the whole linear system in the form $A x-b$ before running the Simplex algorithm. For instance, if we want to lower tighten a function $f(x)$ by expressions (1) and (2) simultaneously, we must rewrite:

1. $f(\underline{x})+\sum_{i} \underline{a_{i}}\left(x_{i}-\underline{x_{i}}\right)=f(\underline{x})+\sum_{i} \underline{a_{i}} x_{i}-\underline{a_{i}} \underline{x_{i}}=\sum_{i} \underline{a_{i}} x_{i}+f(\underline{x})-\sum_{i} \underline{a_{i}} \underline{x_{i}}$

2. $f(\bar{x})+\sum_{i} \overline{a_{i}}\left(x_{i}-\overline{x_{i}}\right)=f(\bar{x})+\sum_{i} \overline{a_{i}} x_{i}-\overline{a_{i} x_{i}}=\sum_{i} \overline{a_{i}} x_{i}+f(\bar{x})-\sum_{i} \overline{a_{i}} \overline{x_{i}}$

Also note that, to remain safe, the computation of constant terms $a_{i} x_{i}$ (resp. $\overline{a_{i}} \overline{x_{i}}$ ) must be achieved with degenerate intervals: $\left[\underline{a_{i}}, \underline{a_{i}}\right]\left[\underline{x_{i}}, \underline{x_{i}}\right]$ (resp. $\left.\left[\overline{a_{i}}, \overline{a_{i}}\right] \overline{\left[\bar{x}_{i}\right.}, \overline{x_{i}}\right]$ ).

\subsection{Preliminary interval linearization}

Recall that the linear forms (1) and (2) proposed by Neumaier and Lin \& Stadtherr use the bounds of the interval gradient, given by $\forall i \in\{1, \ldots, n\},\left[a_{i}\right]=\left[\frac{\partial f}{\partial x_{i}}\right]_{N}([x])$.

Eldon R. Hansen proposed in 1968 a variant in which the Taylor form is achieved recursively, one variable after the other [12,14]. The variant amounts in producing the following tighter interval coefficients:

$$
\forall i \in\{1, \ldots, n\},\left[a_{i}\right]=\left[\frac{\partial f}{\partial x_{i}}\right]_{N}\left(\left[x_{1}\right] \times \ldots \times\left[x_{i}\right] \times x_{i+1} \times \ldots \times \dot{x_{n}}\right)
$$

where $\dot{x_{j}} \in\left[x_{j}\right]$, e.g., $\dot{x_{j}}=m\left(\left[x_{j}\right]\right)$.

By following Hansen's recursive principle, we can produce Hansen's variant of the form (1), for instance, in which the scalar coefficients $\underline{a_{i}}$ are:

$$
\forall i \in\{1, \ldots, n\}, \underline{a_{i}}=\underline{\left[\frac{\partial f}{\partial x_{i}}\right]_{N}\left(\left[x_{1}\right] \times \ldots \times\left[x_{i}\right] \times \underline{x_{i+1}} \times \ldots \times \underline{x_{n}}\right) .}
$$

We end up with an X-Taylor algorithm (X-Taylor stands for eXtremal interval Taylor) producing 2 linear expressions lower tightening a given function $f: \mathbb{R}^{n} \rightarrow \mathbb{R}$ on a given domain $[x]$. The first corner is randomly selected, the second one is opposite to the first one.

\section{4 eXtremal interval Newton}

We first describe in Section 4.1 an algorithm for computing the (box) hull of the polytope produced by X-Taylor. We then detail in Section 4.2 how this X-NewIter procedure is iteratively called in the $\mathrm{X}$-Newton algorithm until a quasi-fixpoint is reached in terms of contraction. 


\subsection{X-Newton iteration}

Algorithm 1 describes a well-known algorithm used in several solvers (see for instance [18, 3]). A specificity here is the use of a corner-based interval Taylor form (X-Taylor) for computing the polytope.

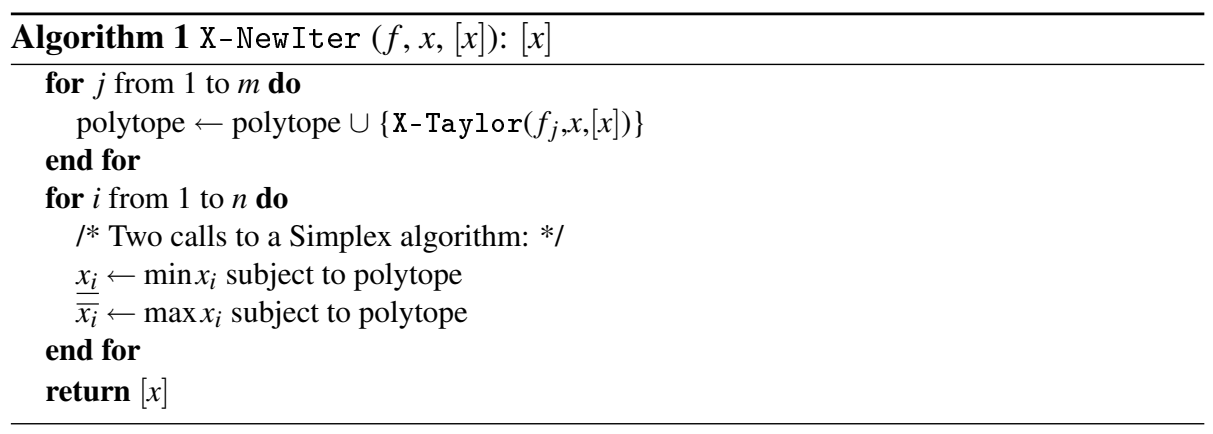

All the constraints appear as inequality constraints $f_{j}(x) \leq 0$ in the vector/set $f=$ $\left(f_{1}, \ldots, f_{j}, \ldots, f_{m}\right) . x=\left(x_{1}, \ldots, x_{i}, \ldots, x_{n}\right)$ denotes the set of variables with domains $[x]$.

The first loop on the constraints builds the polytope while the second loop on the variables contracts the domains, without loss of solution, by calling a Simplex algorithm twice per variable. When embedded in an interval $\mathrm{B} \& \mathrm{~B}$ for constrained global optimization, $\mathrm{X}$-NewIter is modified to also compute a lower bound of the objective in the current box: an additional call to the Simplex algorithm minimizes an X-Taylor relaxation of the objective on the same polytope.

Heuristics mentioned in [3] indicate in which order the variables can be handled, thus avoiding in practice to call $2 n$ times the Simplex algorithm.

\subsection{X-Newton}

The procedure $\mathrm{X}$-NewIter allows one to build the X-Newton operator (see Algorithm 2). Consider first the basic variant in which CP-contractor $=\perp$. X-NewIter

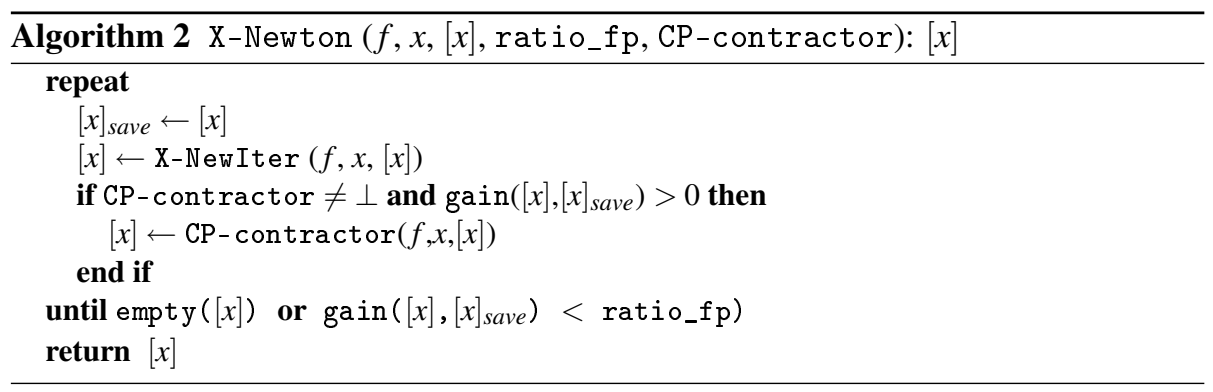

is iteratively run until a quasi fixed-point is reached in terms of contraction. More precisely, ratio_fp is a user-defined percentage of the interval size and:

$$
\operatorname{gain}\left(\left[x^{\prime}\right],[x]\right):=\max _{i} \frac{w\left(\left[x_{i}\right]\right)-w\left(\left[x_{i}^{\prime}\right]\right)}{w\left(\left[x_{i}\right]\right)} .
$$

We also permit the use of a contraction algorithm, typically issued from constraint programming, inside the main loop. For instance, if the user specifies $\mathrm{CP}$-contract or=Mohc 
and if $\mathrm{X}$-NewIter reduces the domain, then the Mohc constraint propagation algorithm [2] can further contract the box, before waiting for the next choice point. The guard gain $\left([x],[x]_{\text {save }}\right)>0$ guarantees that $\mathrm{CP}$-contractor will not be called twice if $\mathrm{X}$-New Iter does not contract the box.

Note that the $X$-Newton operator does not require the system be preconditioned so that this contractor can cut branches early during the tree search (see Section 5.2). In this sense, it is closer to a reliable convexification method like Quad [18, 17] or affine arithmetic [26].

\section{Quadratic convergence}

Compared to a standard interval Newton, a drawback of X-Newton is the loss of quadratic convergence when the current box belongs to a convergence basin. It is however possible to switch from an endpoint Taylor form to a midpoint one and thus be able to obtain quadratic convergence, as detailed in Section $\mathrm{A}$

\section{Existence test for systems of inequalities}

The $X$-Newton operator is a contractor but does not provide any guarantee that a solution exists inside the returned box. Since this operator is adapted to systems of inequality constraints, we detail in Section $B$ an original existence test that applies to this class of systems.

\section{Experiments}

We have applied X-Newton to constrained global optimization and to constraint satisfaction problems.

\subsection{Experiments in constrained global optimization}

We have selected a sample of global optimization systems among those tested by Ninin et al. [26]. They have proposed an interval Branch and Bound, called here IBBA+, that uses constraint propagation and a sophisticated variant of affine arithmetic. From their benchmark of 74 polynomial and non polynomial systems (without trigonometric operators), we have extracted the 27 ones that required more than 1 second to be solved by the simplest version of Ibex0pt (column 4). Table 1 shows the 11 systems solved by this first version in a time comprised between 1 and 11 seconds. Table 2 includes the 13 systems solved in more than 11 seconds 7 Three systems (ex6_2_5, ex6_2_7 and ex6_2_13) are removed from the benchmark because they are not solved by any solver. The reported results have been obtained on a same computer (Intel X86, 3Ghz).

We have implemented the different algorithms in the Interval-Based EXplorer [9] (Ibex). Reference [31] details how our interval B\&B, called IbexOpt, handles constrained optimization problems by using recent and new algorithms. Contraction steps are achieved by the Mohc interval constraint propagation algorithm [2] (that also lower bounds the range of the objective function). The upper bounding phase uses original algorithms for extracting inner regions inside the feasible search space, i.e., zones in

\footnotetext{
${ }^{7}$ Note that most of these systems are also difficult for the non reliable state-of-the-art global optimizer Baron [30], i.e., they are solved in a time comprised between 1 second and more than 1000 seconds (time out).
} 
Table 1: Experimental results on medium difficult global optimization systems

\begin{tabular}{|c|c|c|c|c|c|c|c|c|c|c|c|c|c|}
\hline System & $n$ & No & Rand & $R+R$ & $\mathrm{R}+\mathrm{op}$ & RRRR & Best & Btop & XIter & XNewt & Ibex' & Ibex" & IBBA+ \\
\hline \multirow[t]{2}{*}{ ex2_1_8 } & 24 & TO & 10.50 & 10.27 & 9.32 & 12.29 & $\mathrm{TO}$ & TO & 8.43 & 8.92 & 47.96 & TO & 26.78 \\
\hline & & & 3605 & 2739 & 2444 & 2200 & & & 1068 & 418 & 38988 & & 1916 \\
\hline \multirow[t]{2}{*}{ ex3_1_1 } & 8 & MO & 1.91 & 1.75 & 1.28 & 1.75 & & & 1.24 & 1.87 & $\mathrm{MO}$ & 121 & 116 \\
\hline & & & 2429 & 1877 & 1529 & 1556 & 1851 & 1516 & 676 & 428 & & 36689 & 131195 \\
\hline \multirow[t]{2}{*}{ ex6_1_4 } & 6 & $\mathrm{MO}$ & 1.74 & 1.48 & 1.10 & 1.59 & & & 1.40 & 1.55 & 1.82 & 2.30 & 2.70 \\
\hline & & & 1844 & 1359 & 1069 & 1146 & 1830 & 1097 & 796 & 540 & 4218 & 2215 & 1622 \\
\hline \multirow[t]{2}{*}{ ex6_2_14 } & 4 & 2.16 & 1.74 & 1.68 & 1.58 & 1.79 & & & 1.58 & 1.49 & 44.53 & 65.26 & 208 \\
\hline & & 1421 & 1290 & 1264 & 1247 & 1239 & 1369 & 1237 & 1066 & 742 & 109745 & 104483 & 95170 \\
\hline \multirow[t]{2}{*}{ ex7_2_1 } & 7 & 883 & 1.23 & 1.28 & 1.22 & 1.57 & & & 0.49 & 0.45 & 13.74 & 5.45 & 24.72 \\
\hline & & $1.2 \mathrm{e}+6$ & 1410 & 1314 & 1280 & 1276 & 1636 & 1336 & 260 & 153 & 33478 & 5139 & 8419 \\
\hline \multirow[t]{2}{*}{ ex7_2_6 } & 3 & 10.52 & 9.42 & 6.63 & 1.24 & 3.65 & & & 4.22 & 2.74 & 0.11 & 0.16 & 1.23 \\
\hline & & 71447 & 31601 & 20874 & 3425 & 9412 & $3.7 \mathrm{e}+5$ & $1.2 \mathrm{e}+5$ & 9211 & 4272 & 570 & 436 & 1319 \\
\hline \multirow[t]{2}{*}{ ex7_3_4 } & 12 & 39.08 & 1.11 & 1.33 & 1.28 & 1.56 & & & 1.66 & 2.25 & TO & TO & TO \\
\hline & & 38291 & 818 & 793 & 770 & 685 & 789 & 760 & 441 & 334 & & & \\
\hline \multirow[t]{2}{*}{ ex14_2_1 } & 5 & 7.57 & 1.04 & 1.09 & 0.95 & 1.28 & & & 0.68 & 0.88 & 8.97 & 21.20 & 36.73 \\
\hline & & 7374 & 768 & 689 & 619 & 587 & 749 & 604 & 336 & 198 & 14476 & 22720 & 16786 \\
\hline \multirow[t]{2}{*}{ ex14_2_3 } & 6 & 20.21 & 2.82 & 3.20 & 2.91 & 3.82 & & & 1.75 & 2.62 & 64.22 & 30.81 & TO \\
\hline & & 11557 & 1203 & 1150 & 1081 & 1017 & 1533 & 979 & 525 & 376 & 55347 & 19410 & \\
\hline \multirow[t]{2}{*}{ ex14_2_4 } & 5 & \begin{tabular}{|l|}
0.96 \\
\end{tabular} & 1.09 & 1.33 & 1.04 & 1.35 & & & 0.65 & 1.09 & 35.32 & 36.80 & 128 \\
\hline & & 657 & 588 & 490 & 471 & 437 & 545 & 481 & 229 & 220 & 34240 & 28249 & 30002 \\
\hline \multirow[t]{2}{*}{ ex14_2_6 } & 5 & 1.11 & 1.20 & 1.21 & 1.24 & 1.51 & & & 1.05 & 1.21 & 42.61 & 72.52 & 238 \\
\hline & & 689 & 578 & 459 & 501 & 424 & 578 & 484 & 368 & 234 & 74630 & 32675 & 74630 \\
\hline \multirow[t]{2}{*}{ Sum } & & & 33.80 & 31.25 & 23.16 & 32.16 & & & 23.15 & 25.07 & 147 & 203 & 638 \\
\hline & & & 46134 & 33308 & 14436 & 19979 & & & 14976 & 7915 & 229402 & 208268 & 227948 \\
\hline Gain & & & 1 & 1.02 & 1.71 & 1.03 & & & 1.50 & 1.40 & & & \\
\hline
\end{tabular}

which all points satisfy the inequality and relaxed equality constraints ${ }^{8}$ The cost of any point inside an inner region may improve the upper bound. Also, at each node of the $\mathrm{B} \& \mathrm{~B}$, the $\mathrm{X}$-Taylor algorithm is used to produce hyperplanes for each inequality constraints and the objective function. On the obtained convex polyhedron, two types of tasks can be achieved: either the lower bounding of the cost with one call to a Simplex algorithm (results reported in columns 4 to 13), or the lower bounding and the contraction of the box, with X-NewIter (i.e., $2 n+1$ calls to a Simplex algorithm; results reported in column 10) or X-Newton (columns 11,13). The bisection heuristic is a variant of Kearfott's Smear function described in [31].

The first two columns contain the name of the handled system and its number of variables. Each entry contains generally the CPU time in second (first line of a multiline) and the number of branching nodes (second line). The same precision on the cost $(1 . e-8)$ and the same timeout (TO $=1$ hour) have been used by Ibex0pt and IBBA+ 9 Cases of memory overflow (MO) sometimes occur. For each method $m$, the last line includes an average gain on the different systems. For a given system, the gain w.r.t. the basic method (column 4) is $\frac{\text { CPUtime }(\text { Rand })}{\text { CPUtime }(m)}$. The last 10 columns of Table 2 compare different variants of X-Taylor and X-Newton. The differences between variants are clearer on the most difficult instances. All use Hansen's variant to compute the interval gradient (see Section 3.2). The gain is generally small but

\footnotetext{
${ }^{8}$ An equation $h_{j}(x)=0$ is relaxed by two inequality constraints: $-\varepsilon \leq h_{j}(x) \leq+\varepsilon$.

${ }^{9}$ The results obtained by IBBA+ on a similar computer are taken from [26].
} 
Hansen's variant is more robust: for instance ex_7_2_3 cannot be solved with the basic interval gradient calculation.

In the column 3 , the convexification operator is removed from our interval $\mathrm{B} \& \mathrm{~B}$, which underlines its significant benefits in practice. The column 4 corresponds to an

Table 2: Experimental results on difficult constrained global optimization systems

\begin{tabular}{|c|c|c|c|c|c|c|c|c|c|c|c|c|c|}
\hline & 2 & 3 & 4 & 5 & 6 & 7 & 8 & 9 & 10 & 11 & 12 & 13 & 14 \\
\hline System & $\mathrm{n}$ & No & Rand & $\mathrm{R}+\mathrm{R}$ & R+op & RRRR & Best & Btop & XIter & XNewt & Ibex' & Ibex" & IBBA+ \\
\hline ex2_1_7 & 20 & TO & $\begin{array}{r}42.96 \\
20439\end{array}$ & $\begin{array}{r}43.17 \\
16492\end{array}$ & $\begin{array}{r}40.73 \\
15477\end{array}$ & $\begin{array}{r}49.48 \\
13200\end{array}$ & TO & TO & $\begin{array}{r}\mathbf{7 . 7 4} \\
1344\end{array}$ & $\begin{array}{r}10.58 \\
514\end{array}$ & TO & TO & $\begin{array}{r}16.75 \\
1574\end{array}$ \\
\hline ex2_1_9 & 10 & MO & $\begin{array}{r}40.09 \\
49146\end{array}$ & $\begin{array}{r}29.27 \\
30323\end{array}$ & $\begin{array}{r}22.29 \\
23232\end{array}$ & $\begin{array}{r}24.54 \\
19347\end{array}$ & 57560 & 26841 & $\begin{array}{r}\mathbf{9 . 0 7} \\
5760\end{array}$ & $\begin{array}{r}9.53 \\
1910\end{array}$ & $\begin{array}{r}46.58 \\
119831\end{array}$ & $\begin{array}{r}103 \\
100987\end{array}$ & $\begin{array}{r}154.02 \\
60007\end{array}$ \\
\hline ex6_1_1 & 8 & MO & $\begin{array}{r}20.44 \\
21804\end{array}$ & $\begin{array}{r}19.08 \\
17104\end{array}$ & $\begin{array}{r}\mathbf{1 7 . 2 3} \\
14933\end{array}$ & $\begin{array}{r}22.66 \\
14977\end{array}$ & 24204 & 15078 & $\begin{array}{r}31.24 \\
14852\end{array}$ & \begin{tabular}{r|}
38.59 \\
13751
\end{tabular} & TO & $\begin{array}{r}633 \\
427468\end{array}$ & TO \\
\hline x6_1_3 & 12 & TO & $\begin{array}{r}1100 \\
522036\end{array}$ & $\begin{array}{r}711 \\
269232\end{array}$ & $\begin{array}{r}529 \\
205940\end{array}$ & \begin{tabular}{|r|}
794 \\
211362
\end{tabular} & TO & TO & $\begin{array}{r}262.5 \\
55280\end{array}$ & \begin{tabular}{r|}
$\mathbf{2 1 9}$ \\
33368
\end{tabular} & TO & TO & TO \\
\hline ex6_2_6 & 3 & TO & $\begin{array}{r}162 \\
172413\end{array}$ & $\begin{array}{r}175 \\
68435\end{array}$ & $\begin{array}{r}169 \\
163076\end{array}$ & $\begin{array}{r}207 \\
163967\end{array}$ & $1.7 \mathrm{e}+5$ & $1.6 \mathrm{e}+5$ & $\begin{array}{r}172 \\
140130\end{array}$ & $\begin{array}{r}\mathbf{1 3 6} \\
61969\end{array}$ & $\begin{array}{r}1033 \\
1.7 \mathrm{e}+6\end{array}$ & $\begin{array}{r}583 \\
770332\end{array}$ & $\begin{array}{r}1575 \\
922664\end{array}$ \\
\hline ex6_2_8 & 3 & $\begin{array}{l}97.10 \\
1.2 \mathrm{e}+5\end{array}$ & $\begin{array}{r}121 \\
117036 \\
\end{array}$ & $\begin{array}{r}119 \\
105777\end{array}$ & $\begin{array}{r}110 \\
97626\end{array}$ & $\begin{array}{r}134.7 \\
98897\end{array}$ & $1.2 \mathrm{e}+5$ & 97580 & $\begin{array}{r}78.1 \\
61047\end{array}$ & $\begin{array}{r}\mathbf{5 9 . 3} \\
25168\end{array}$ & \begin{tabular}{|r}
284 \\
523848
\end{tabular} & $\begin{array}{r}274 \\
403668\end{array}$ & $\begin{array}{r}458 \\
265276\end{array}$ \\
\hline ex6_2_9 & 4 & $\begin{array}{r}\mathbf{2 5 . 2 0} \\
27892\end{array}$ & $\begin{array}{r}33.0 \\
27892\end{array}$ & $\begin{array}{r}36.7 \\
27826\end{array}$ & $\begin{array}{r}35.82 \\
27453\end{array}$ & $\begin{array}{r}44.68 \\
27457\end{array}$ & 27881 & 27457 & $\begin{array}{r}42.34 \\
27152\end{array}$ & $\begin{array}{r}43.74 \\
21490\end{array}$ & $\begin{array}{r}455 \\
840878\end{array}$ & $\begin{array}{r}513 \\
684302\end{array}$ & $\begin{array}{r}523 \\
203775\end{array}$ \\
\hline ex6_2_10 & 6 & TO & $\begin{array}{r}3221 \\
1.6 \mathrm{e}+6\end{array}$ & $\begin{array}{r}2849 \\
1.2 \mathrm{e}+6\end{array}$ & $\begin{array}{r}\mathbf{1 9 2 4} \\
820902 \\
\end{array}$ & $\begin{array}{r}2905 \\
894893 \\
\end{array}$ & $1.1 \mathrm{et} 6$ & $8.2 \mathrm{e}+5$ & \begin{tabular}{|r|}
2218 \\
818833
\end{tabular} & \begin{tabular}{|r|}
2697 \\
656360 \\
\end{tabular} & TO & TO & TO \\
\hline ex6_2_11 & 3 & $\begin{array}{r}10.57 \\
17852\end{array}$ & $\begin{array}{r}19.31 \\
24397\end{array}$ & $\begin{array}{r}\mathbf{7 . 5 1} \\
8498\end{array}$ & $\begin{array}{r}7.96 \\
8851\end{array}$ & $\begin{array}{r}10.82 \\
10049\end{array}$ & 5606 & 27016 & $\begin{array}{r}13.26 \\
12253\end{array}$ & $\begin{array}{r}11.08 \\
6797\end{array}$ & $\begin{array}{r}41.21 \\
93427\end{array}$ & $\begin{array}{r}11.80 \\
21754\end{array}$ & $\begin{array}{r}140.51 \\
83487\end{array}$ \\
\hline ex6_2_12 & 4 & $\begin{array}{r}2120 \\
2 \mathrm{e}+6\end{array}$ & $\begin{array}{r}232 \\
198156\end{array}$ & $\begin{array}{r}160 \\
13893\end{array}$ & $\begin{array}{r}118.6 \\
86725\end{array}$ & $\begin{array}{r}155 \\
90414\end{array}$ & $1.9 \mathrm{e}+5$ & 86729 & $\begin{array}{r}51.31 \\
31646\end{array}$ & $\begin{array}{r}\mathbf{2 2 . 2 0} \\
7954\end{array}$ & $\begin{array}{r}122 \\
321468\end{array}$ & $\begin{array}{r}187 \\
316675\end{array}$ & $\begin{array}{r}112.58 \\
58231\end{array}$ \\
\hline ex7_3_5 & 13 & TO & $\begin{array}{r}44.7 \\
45784 \\
\end{array}$ & $\begin{array}{r}54.9 \\
44443 \\
\end{array}$ & $\begin{array}{r}60.3 \\
50544 \\
\end{array}$ & $\begin{array}{r}75.63 \\
43181 \\
\end{array}$ & 45352 & 42453 & $\begin{array}{r}29.88 \\
6071 \\
\end{array}$ & $\begin{array}{r}\mathbf{2 8 . 9 1} \\
5519 \\
\end{array}$ & TO & TO & TO \\
\hline ex14_1_7 & 10 & TO & $\begin{array}{r}433 \\
223673 \\
\end{array}$ & $\begin{array}{r}445 \\
172671 \\
\end{array}$ & $\begin{array}{r}\mathbf{4 0 6} \\
156834 \\
\end{array}$ & $\begin{array}{r}489 \\
125121 \\
\end{array}$ & $1.7+5$ & $1.1+5$ & $\begin{array}{r}786 \\
179060 \\
\end{array}$ & $\begin{array}{r}938 \\
139111 \\
\end{array}$ & TO & TO & TO \\
\hline ex14_2_7 & 6 & $\begin{array}{r}93.10 \\
35517\end{array}$ & $\begin{array}{r}94.16 \\
25802\end{array}$ & $\begin{array}{r}102.2 \\
21060\end{array}$ & $\begin{array}{r}83.6 \\
16657\end{array}$ & $\begin{array}{r}113.7 \\
15412\end{array}$ & 20273 & 18126 & $\begin{array}{r}\mathbf{6 6 . 3 9} \\
12555\end{array}$ & $\begin{array}{r}97.36 \\
9723\end{array}$ & TO & TO & TO \\
\hline Sum & & & $\begin{array}{r}5564 \\
3.1 \mathrm{e}+6\end{array}$ & $\begin{array}{r}4752 \\
2.2 \mathrm{e}+6\end{array}$ & $\begin{array}{r}\mathbf{3 5 2 5} \\
1.7 \mathrm{e}+6\end{array}$ & $\begin{array}{r}5026 \\
1.7 \mathrm{e}+6\end{array}$ & & & $\begin{array}{r}3767 \\
1.4 \mathrm{e}+6\end{array}$ & $\begin{array}{r}4311 \\
983634\end{array}$ & $\begin{array}{r}1982 \\
3.6 \mathrm{e}+6\end{array}$ & $\begin{array}{r}1672 \\
2.3 \mathrm{e}+6\end{array}$ & $\begin{array}{r}2963 \\
1.6 \mathrm{e}+6\end{array}$ \\
\hline Gain & & & 1 & 1.21 & 1.39 & 1.07 & & & 2.23 & 1.78 & & & \\
\hline ex7_2_3 & 8 & MO & MO & MO & MO & MO & & & $\begin{array}{r}\mathbf{5 4 4} \\
611438 \\
\end{array}$ & \begin{tabular}{|r|}
691 \\
588791 \\
\end{tabular} & TO & $\begin{array}{r}719 \\
681992 \\
\end{array}$ & TO \\
\hline
\end{tabular}

$\mathrm{X}$-Taylor performed with one corner randomly picked for every constraint. The next column $(\mathrm{R}+\mathrm{R})$ corresponds to a tighter polytope computed with two randomly chosen corners per inequality constraint. The gain is small w.r.t. Rand. The column 6 (R+op) highlights the best $\mathrm{X}$-Taylor variant where a random corner is chosen along with its opposite corner. Working with more than 2 corners appeared to be counter-productive, as shown by the column 7 (RRRR) that corresponds to 4 corners randomly picked.

We have performed a very informative experiment whose results are shown in columns 8 (Best) and $9(B+o p)$ : an exponential algorithm selects the best corner, maximizing the expression (4), among the $2^{n}$ ones 10 The reported number of branching nodes shows that the best corner (resp. $B+o p$ ) sometimes brings no additional contraction and often brings a very small one w.r.t. a random corner (resp. $R+o p$ ). Therefore, the combination $R+o p$ has been kept in all the remaining variants (columns 10 to 14).

The column 10 (XIter) reports the results obtained by X-NewIter. It shows the best performance on average while being robust. In particular, it avoids the memory

\footnotetext{
${ }^{10}$ We could not thus compute the number of branching nodes of systems with more than 12 variables because they reached the timeout.
} 
overflow on ex7_2_3. X-Newton, using ratio_f $\mathrm{p}=20 \%$, is generally slightly worse, although a good result is obtained on ex6_2_12 (see column 11).

The last three columns report a first comparison between AA (affine arithmetic; Ninin et al.'s AF2 variant) and our convexification methods. Since we did not encode AA in our solver due to the significant development time required, we have transformed Ibex0pt into two variants Ibex' and Ibex" very close to IBBA+: Ibex' and Ibex" use a non incremental version of HC4 [5] that loops only once on the constraints, and a largest-first branching strategy. The upper bounding is also the same as IBBA+ one. Therefore we guess that only the convexification method differs from IBBA+: Ibex, improves the lower bound using a polytope based on a random corner and its opposite corner; Ibex"' builds the same polytope but uses X-Newt on to better contract on all the dimensions 11

First, Ibex' reaches the timeout once more than IBBA+; and IBBA+ reaches the timeout once more than Ibex". Second, the comparison in the number of branching points (the line Sum accounts only the systems that the three strategies solve within the timeout) underlines that AA contracts generally more than Ibex', but the difference is smaller with the more contracting Ibex"' (that can also solve ex7_2_3). This suggests that the job on all the variables compensates the relative lack of contraction of $\mathrm{X}$-Taylor. Finally, the performances of Ibex' and Ibex' are better than IBBA+ one, but it is probably due to the different implementations.

\subsection{Experiments in constraint satisfaction}

We have also tested the $\mathrm{X}$-Newt on contractor in constraint satisfaction, i.e., for solving well constrained systems having a finite number of solutions. These systems are generally square systems ( $n$ equations and $n$ variables). The constraints correspond to non linear differentiable functions (some systems are polynomial, others are not). We have selected from the COPRIN benchmark ${ }^{12}$ all the systems that can be solved by one of the tested algorithms in a time between $10 \mathrm{~s}$ and $1000 \mathrm{~s}$ : we discarded easy problems solved in less than 10 seconds, and too difficult problems that no method can solve in less than 1000 seconds. The timeout was fixed to one hour. The required precision on the solution is $10^{-8}$. Some of these problems are scalable. In this case, we selected the problem with the greatest size (number of variables) that can be solved by one of the tested algorithms in less than 1000 seconds.

We compared our method with the state of art algorithm for solving such problems in their original form (we did not use rewriting of constraints and did not exploit common subexpressions). We used as reference contractor our best contractor $\operatorname{ACID}($ Mohc), an adaptive version of CID [32] with Mohc [2] as basic contractor, that exploits the monotonicity of constraints. We used the same bisection heuristic as in optimization experiments. Between two choice points in the search tree, we called one of the following contractors (see Table 3 ).

- $\operatorname{ACID}($ Mohc) : see column 3 (Ref),

- X-New Iter: ACID (Mohc) followed by one call to Algorithm 1 (column 4, Xiter),

- X-Newton: the most powerful contractor with ratio_fp=20\%, and ACID (Mohc) as internal CP contractor (see Algorithm 2).

\footnotetext{
${ }^{11}$ We have removed the call to Mohc inside the $\mathrm{X}$-Newt on loop (i.e., CP-contractor $=\perp$ ) because this constraint propagation algorithm is not a convexification method.

12 http: //www-sop.inria.fr/coprin/logiciels/ALIAS/Benches/benches .html
} 
For X-Newton, we have tested 5 ways for selecting the corners (see columns 5-9):

- Rand: one random corner,

- R+R: two random corners,

- R+op: one random corner and its opposite,

- RRRR: four random corners,

- 2R+op: four corners, i.e., two random corners and their two respective opposite ones.

We can observe that, as for the optimization problems, the corner selection R+op yields the lowest sum of solving times and often good results. The performance profile 2 (and the last line of Table 3) highlights that all the 24 systems can be solved in $1000 \mathrm{~s}$ by $\mathrm{X}$-Newton $\mathrm{R}+\mathrm{op}$, while only 18 systems are solved in $1000 \mathrm{~s}$ by the reference algorithm with no convexification method. Each entry in Table 3 contains the CPU

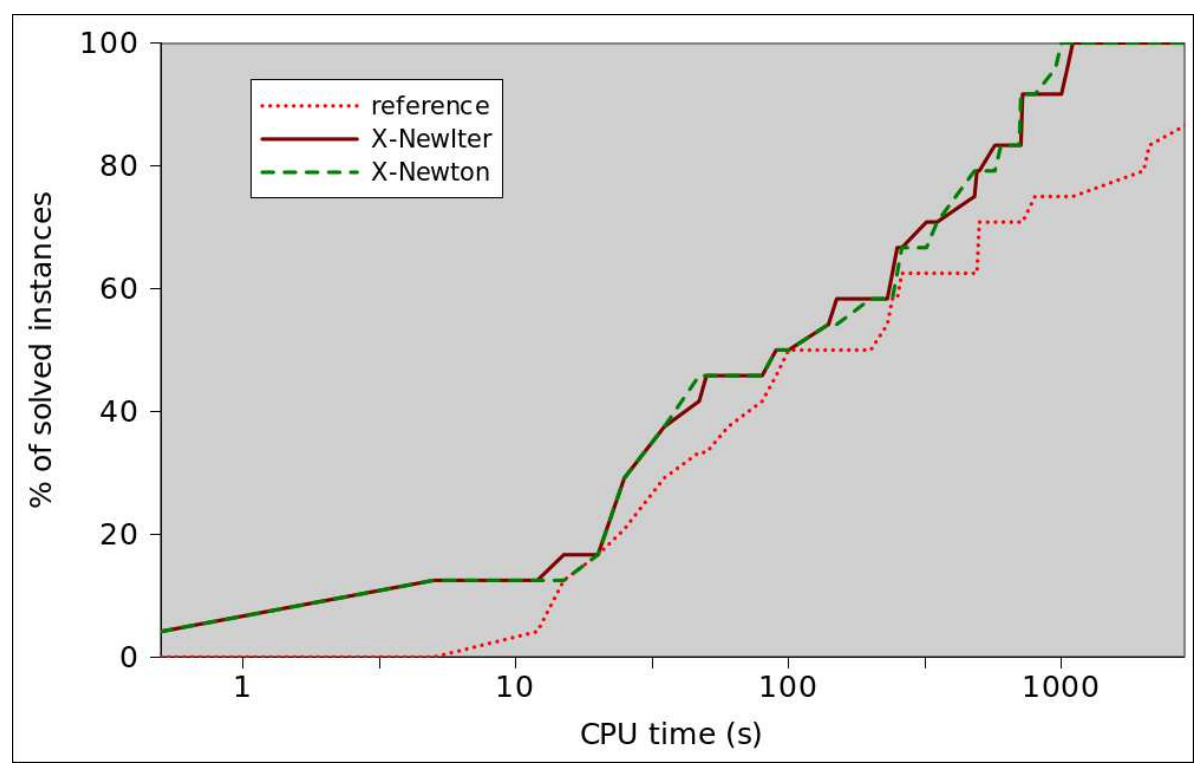

Figure 2: Performance profile. The curves show, for a given algorithm, the percentage of systems solved as a function of the CPU time in second.

time in second (first line of a multi-line) and the number of branching nodes (second line). We have reported in the last column (Gain) the gains obtained by the best corner selection strategy $\mathrm{R}+\mathrm{op}$ as the ratio w.r.t. the reference method (column $3 \mathrm{Ref}$ ), i.e., $\frac{C P U \text { time }(R+o p)}{C P U \text { time }(\operatorname{Ref})}$. Note that we used the inverse gain definition compared to the one used in optimization (see 5.1) in order to manage the problems reaching the timeout. We can also observe that our new algorithm $\mathrm{X}$-Newton $\mathrm{R}+\mathrm{op}$ is efficient and robust: we can obtain significant gains (small values in bold) and lose never more than $39 \%$ in CPU time.

We have finally tried, for the scalable systems, to solve problems of bigger size. We could solve Katsura-30 in 4145 s, and Yamamura1-16 in 2423 s (instead of $33521 \mathrm{~s}$ with the reference algorithm). We can remark that, for these problems, the gain grows with the size. 
Table 3: Experimental results on difficult constraint satisfaction problems. The best results and the gains $(<1)$ appear in bold.

\begin{tabular}{|c|c|c|c|c|c|c|c|c|c|}
\hline 1 & 2 & 3 & 4 & 5 & 6 & 7 & 8 & 9 & 10 \\
\hline System & $\mathrm{n}$ & Ref & Xiter & Rand & $\mathrm{R}+\mathrm{R}$ & $\mathrm{R}+\mathrm{op}$ & RRRR & $2 \mathrm{R}+\mathrm{op}$ & Gain \\
\hline \multirow[t]{2}{*}{ Bellido } & \multirow[t]{2}{*}{9} & 10.04 & 3.88 & 4.55 & 3.71 & 3.33 & 3.35 & 3.28 & \multirow[t]{2}{*}{0.33} \\
\hline & & 3385 & 1273 & 715 & 491 & 443 & 327 & 299 & \\
\hline \multirow[t]{2}{*}{ Bratu-60 } & \multirow[t]{2}{*}{60} & 494 & 146 & 306 & 218 & 190 & 172 & 357 & \multirow[t]{2}{*}{0.38} \\
\hline & & 9579 & 3725 & 4263 & 3705 & 3385 & 3131 & 5247 & \\
\hline \multirow[t]{2}{*}{ Brent-10 } & \multirow[t]{2}{*}{10} & 25.31 & 28 & 31.84 & 33.16 & 34.88 & 37.72 & 37.11 & \multirow[t]{2}{*}{1.38} \\
\hline & & 4797 & 4077 & 3807 & 3699 & 3507 & 3543 & 3381 & \\
\hline \multirow[t]{2}{*}{ Brown-10 } & \multirow[t]{2}{*}{10} & \multirow[t]{2}{*}{ TO } & 0.13 & 0.17 & 0.17 & 0.17 & 0.17 & 0.18 & \multirow[t]{2}{*}{$\mathbf{0}$} \\
\hline & & & 67 & 49 & 49 & 49 & 49 & 49 & \\
\hline \multirow[t]{2}{*}{ Butcher8-a } & \multirow[t]{2}{*}{8} & 233 & 246 & 246 & 248 & 242 & 266 & 266 & \multirow[t]{2}{*}{1.06} \\
\hline & & 40945 & 39259 & 36515 & 35829 & 35487 & 33867 & 33525 & \\
\hline \multirow[t]{2}{*}{ Butcher8-b } & 8 & 97.9 & 123 & 113.6 & 121.8 & 122 & 142.4 & 142.2 & 1.26 \\
\hline & & 26693 & 23533 & 26203 & 24947 & 24447 & 24059 & 24745 & \\
\hline Design & 9 & 21.7 & 23.61 & 22 & 22.96 & 22.38 & 25.33 & 25.45 & 1.03 \\
\hline & & 3301 & 3121 & 2793 & 2549 & 2485 & 2357 & 2365 & \\
\hline Direct Kinematics & 11 & 85.28 & 81.25 & 84.96 & 83.52 & 84.28 & 86.15 & 85.62 & 0.99 \\
\hline & & 1285 & 1211 & 1019 & 929 & 915 & 815 & 823 & \\
\hline Dietmaier & 12 & 3055 & 1036 & 880 & 979 & 960 & 1233 & 1205 & 0.31 \\
\hline & & 493957 & 152455 & 113015 & 96599 & 93891 & 85751 & 83107 & \\
\hline Discrete integral-16 & 32 & $\mathrm{TO}$ & 480 & 469 & 471 & 472 & 478 & 476 & $\mathbf{0}$ \\
\hline 2nd form. & & & 57901 & 57591 & 57591 & 57591 & 57591 & 57591 & \\
\hline Eco9 & 8 & 12.85 & 14.19 & 14.35 & 14.88 & 15.05 & 17.48 & 17.3 & 1.17 \\
\hline & & 4573 & 3595 & 3491 & 2747 & 2643 & 2265 & 2159 & \\
\hline Ex14-2-3 & 6 & 45.01 & 3.83 & 4.39 & 3.88 & 3.58 & 3.87 & 3.68 & 0.08 \\
\hline & & 3511 & 291 & 219 & 177 & 181 & 145 & 139 & \\
\hline Fredtest & 6 & 74.61 & 47.73 & 54.46 & 47.43 & 44.26 & 42.67 & 40.76 & 0.59 \\
\hline & & 18255 & 12849 & 11207 & 8641 & 7699 & 6471 & 6205 & \\
\hline Fourbar & 4 & 258 & 317 & 295 & 319 & 320 & 366 & 367 & 1.24 \\
\hline & & 89257 & 83565 & 79048 & 73957 & 75371 & 65609 & 67671 & \\
\hline Geneig & 6 & 57.32 & 46.1 & 46.25 & 41.33 & 40.38 & 38.4 & 38.43 & 0.7 \\
\hline & & 3567 & 3161 & 2659 & 2847 & 2813 & 2679 & 2673 & \\
\hline I5 & 10 & 17.21 & 20.59 & 19.7 & 20.53 & 20.86 & 23.23 & 23.43 & 1.21 \\
\hline & & 5087 & 4931 & 5135 & 4885 & 4931 & 4843 & 4861 & \\
\hline Katsura-25 & 26 & TO & 711 & 1900 & 1258 & 700 & 1238 & 1007 & $\mathbf{0}$ \\
\hline & & & 9661 & 17113 & 7857 & 4931 & 5013 & 4393 & \\
\hline Pramanik & 3 & 14.69 & 20.08 & 19.16 & 20.31 & 20.38 & 24.58 & 25.15 & 1.39 \\
\hline & & 18901 & 14181 & 14285 & 11919 & 11865 & 11513 & 12027 & \\
\hline Synthesis & 33 & 212 & 235 & 264 & 316 & 259 & 631 & 329 & 1.22 \\
\hline & & 9097 & 7423 & 7135 & 6051 & 4991 & 7523 & 3831 & \\
\hline Trigexp2-17 & 17 & 492 & 568 & 533 & 570 & 574 & 630 & 637 & 1.17 \\
\hline & & 27403 & 27049 & 26215 & 25805 & 25831 & 25515 & 25055 & \\
\hline Trigo1-14 & 14 & 2097 & 1062 & 1314 & 1003 & 910 & 865 & 823 & 0.43 \\
\hline & & 8855 & 5229 & 4173 & 2773 & 2575 & 1991 & 1903 & \\
\hline Trigonometric & 5 & 33.75 & 30.99 & 30.13 & 30.11 & 30.65 & 31.13 & 31.75 & 0.91 \\
\hline & & 4143 & 3117 & 2813 & 2265 & 2165 & 1897 & 1845 & \\
\hline Virasoro & 8 & 760 & 715 & 729 & 704 & 709 & 713 & 715 & 0.93 \\
\hline & & 32787 & 35443 & 33119 & 32065 & 32441 & 30717 & 27783 & \\
\hline Yamamura1-14 & 14 & 1542 & 407 & 628 & 557 & 472 & 520 & 475 & 0.26 \\
\hline & & 118021 & 33927 & 24533 & 23855 & 14759 & 13291 & 11239 & \\
\hline Sum & & $>42353$ & 6431 & 8000 & 7087 & 6185 & 7588 & 7131 & \\
\hline & & $>1.8 \mathrm{e} 6$ & 531044 & 477115 & 432232 & 415396 & 382862 & 382916 & \\
\hline Gain & & 1 & 0.75 & 0.77 & 0.78 & 0.76 & 0.9 & 0.85 & \\
\hline Solved in $1000 \mathrm{~s}$ & & 18 & 22 & 22 & 22 & 24 & 22 & 22 & \\
\hline
\end{tabular}




\section{Conclusion}

Endowing a solver with a reliable convexification algorithm is useful in constraint satisfaction and crucial in constrained global optimization. This paper has presented the probably simplest way to produce a reliable convexification of the solution space and the objective function. X-Taylor can be encoded in 100 lines of codes and calls a standard Simplex algorithm. It rapidly computes a polyhedral convex relaxation following Hansen's recursive principle to produce the gradient and using two corners as expansion point of Taylor: a corner randomly selected and the opposite corner.

This convex interval Taylor form can be used to build an eXtremal interval Newton. The X-NewIter variant contracting all the variable intervals once provides on average the best performance on constrained global optimization systems. For constraint satisfaction, both algorithms yield comparable results.

Compared to affine arithmetic, preliminary experiments suggest that our convex interval Taylor produces a looser relaxation in less CPU time. However, the additional job achieved by X-Newton can compensate this lack of filtering at a low cost, so that one can solve one additional tested system in the end. Therefore, we think that this reliable convexification method has the potential to complement affine arithmetic and Quad.

\section{Acknowledgment}

We would like to particularly thank G. Chabert for useful discussions about existing interval analysis results.

\section{References}

[1] O. Aberth. The Solution of Linear Interval Equations by a Linear Programming Method. Linear Algebra and its Applications, 259:271-279, 1997.

[2] I. Araya, G. Trombettoni, and B. Neveu. Exploiting Monotonicity in Interval Constraint Propagation. In Proc. AAAI, pages 9-14, 2010.

[3] A. Baharev, T. Achterberg, and E. Rév. Computation of an Extractive Distillition Column with Affine Arithmetic. AIChE Journal, 55(7):1695-1704, 2009.

[4] O. Beaumont. Algorithmique pour les intervalles. $\mathrm{PhD}$ thesis, Université de Rennes, 1997.

[5] F. Benhamou, F. Goualard, L. Granvilliers, and J.-F. Puget. Revising Hull and Box Consistency. In Proc. ICLP, pages 230-244, 1999.

[6] C. Bliek. Computer Methods for Design Automation. PhD thesis, MIT, 1992.

[7] G. Chabert. Techniques d'intervalles pour la résolution de systèmes d'intervalles. $\mathrm{PhD}$ thesis, Université de Nice-Sophia, 2007.

[8] G. Chabert and N. Beldiceanu. Sweeping with Continuous Domains. In Proc. CP, LNCS 6308, pages 137-151, 2010.

[9] G. Chabert and L. Jaulin. Contractor Programming. Artificial Intelligence, 173:1079-1100, 2009. 
[10] L. de Figueiredo and J. Stolfi. Affine Arithmetic: Concepts and Applications. Numerical Algorithms, 37(1-4):147-158, 2004.

[11] A. Goldsztejn and L. Granvilliers. A New Framework for Sharp and Efficient Resolution of NCSP with Manifolds of Solutions. Constraints (Springer), 15(2):190$212,2010$.

[12] E.R. Hansen. On Solving Systems of Equations Using Interval Arithmetic. Mathematical Comput., 22:374-384, 1968.

[13] E.R. Hansen. Bounding the Solution of Interval Linear Equations. SIAM J. Numerical Analysis, 29(5):1493-1503, 1992.

[14] E.R. Hansen. Global Optimization using Interval Analysis. Marcel Dekker inc., 1992.

[15] R. B. Kearfott. Rigorous Global Search: Continuous Problems. Kluwer Academic Publishers, 1996.

[16] V. Kreinovich, A.V. Lakeyev, J. Rohn, and P.T. Kahl. Computational Complexity and Feasibility of Data Processing and Interval Computations. Kluwer, 1997.

[17] Y. Lebbah, C. Michel, and M. Rueher. An Efficient and Safe Framework for Solving Optimization Problems. J. Computing and Applied Mathematics, 199:372377, 2007.

[18] Y. Lebbah, C. Michel, M. Rueher, D. Daney, and J.P. Merlet. Efficient and safe global constraints for handling numerical constraint systems. SIAM Journal on Numerical Analysis, 42(5):2076-2097, 2005.

[19] Y. Lin and M. Stadtherr. LP Strategy for the Interval-Newton Method in Deterministic Global Optimization. Industrial \& engineering chemistry research, 43:3741-3749, 2004.

[20] D. McAllester, P. Van Hentenryck, and D. Kapur. Three Cuts for Accelerated Interval Propagation. Technical Report AI Memo 1542, Massachusetts Institute of Technology, 1995.

[21] F. Messine, , and J.-L. Laganouelle. Enclosure Methods for Multivariate Differentiable Functions and Application to Global Optimization. Journal of Universal Computer Science, 4(6):589-603, 1998.

[22] R. E. Moore. Interval Analysis. Prentice-Hall, 1966.

[23] R.E. Moore, R. B. Kearfott, and M.J. Cloud. Introduction to Interval Analysis. SIAM, 2009.

[24] A. Neumaier. Interval Methods for Systems of Equations. Cambridge Univ. Press, 1990.

[25] A. Neumaier and O. Shcherbina. Safe Bounds in Linear and Mixed-Integer Programming. Mathematical Programming, 99:283-296, 2004.

[26] J. Ninin, F. Messine, and P. Hansen. A Reliable Affine Relaxation Method for Global Optimization. Submitted (research report RT-APO-10-05, IRIT, march 2010), 2010. 
[27] W. Oettli. On the Solution Set of a Linear System with Inaccurate Coefficients. SIAM J. Numerical Analysis, 2(1):115-118, 1965.

[28] J. Rohn. Cheap and Tight Bounds: The Recent Result by E. Hansen can be Made More Efficient. Interval Computations, 1:13-21, 1993.

[29] T. J. Schaefer. The Complexity of Satisfiability Problems. In Proc. STOC, ACM symposium on theory of computing, pages 216-226, 1978.

[30] M. Tawarmalani and N. V. Sahinidis. A Polyhedral Branch-and-Cut Approach to Global Optimization. Mathematical Programming, 103(2):225-249, 2005.

[31] G. Trombettoni, I. Araya, B. Neveu, and G. Chabert. Inner Regions and Interval Linearizations for Global Optimization. In AAAI, pages 99-104, 2011.

[32] G. Trombettoni and G. Chabert. Constructive Interval Disjunction. In Proc. CP, LNCS 4741, pages 635-650, 2007.

[33] X.-H. Vu, D. Sam-Haroud, and B. Faltings. Enhancing Numerical Constraint Propagation using Multiple Inclusion Representations. Annals of Mathematics and Artificial Intelligence, 55(3-4):295-354, 2009. 


\section{A X-Newton and square systems of equations}

In the case where square constraint systems are handled, the standard interval Newton operator, called I-Newt on hereafter, sometimes detects cases where the system falls in a convergence basin and obtains quadratic convergence. The X-Newt on operator cannot have the same property a priori, but we propose a hybrid version Square-X-Newton of $\mathrm{X}$-Newton that can switch from a endpoint Taylor form to a midpoint one when a necessary condition holds. (The possibility of calling CP-contractor is not considered in this section for the sake of clarity.)

Let us first recall the principle of the standard interval Newton operator.

\section{A.1 Standard interval Newton}

We consider here that $f=\left(f_{1}, \ldots, f_{j}, \ldots, f_{m}\right)$ is the set of functions involved in the set of equations $f_{j}(x)=0$ handled by the algorithm. Let $x$ be a vector of variables and $[x]=\left[x_{1}\right] \times \ldots \times\left[x_{i}\right] \times \ldots \times\left[x_{n}\right]$ its domain. Let $[A]$ be the interval Jacobian matrix (Hansen's variant) obtained with a midpoint interval Taylor form, i.e., a matrix in which every element is the interval:

$$
\left[a_{i, j}\right]=\left[\frac{\partial f_{j}}{\partial x_{i}}\right]_{N}\left(\left[x_{1}\right] \times \ldots \times\left[x_{i}\right] \times m\left(\left[x_{i+1}\right]\right) \times \ldots \times m\left(\left[x_{n}\right]\right) .\right.
$$

One iteration of the interval Newton operator contracts the current box. It returns a box $\left[x^{\prime}\right]$ and intersects it with the current box $[x]$, as follows:

1. Compute the Jacobian matrix $[A]$ of $f$ in $[x]$ with a midpoint interval Taylor form.

Compute the vector of values $b:=-f(m([x])$.

2. Compute $P:=m([A])^{-1}$.

3. Preconditioning: $\left[A^{\prime}\right]:=P .[A] ; b^{\prime}:=P . b$.

4. Compute the hull $\left[x^{\prime}\right]$ of the solution set of the interval linear system: $\left[A^{\prime}\right][x]=b^{\prime}$.

5. $[x]:=[x] \cap\left[x^{\prime}\right]$

Several such iterations are launched until a quasi fixed-point is reached in terms of contraction.

The step 4 of an interval Newton iteration can be performed by several methods, such as an interval Gauss-Seidel or the Hansen-Bliek method mentioned above [6, 14. 28]. Also, if after step 4 we have $\left[x^{\prime}\right] \subseteq[x]$, then it is guaranteed that a unique solution exists inside $\left[x^{\prime}\right]$ and that further iterations will quadratically converge to this solution [24].

\section{A.2 I-Newton and X-Newton for square systems}

In the case where square constraint systems are handled, X-Newton can be specialized to a Square-X-Newton variant that can theoretically obtain sometimes quadratic convergence. 


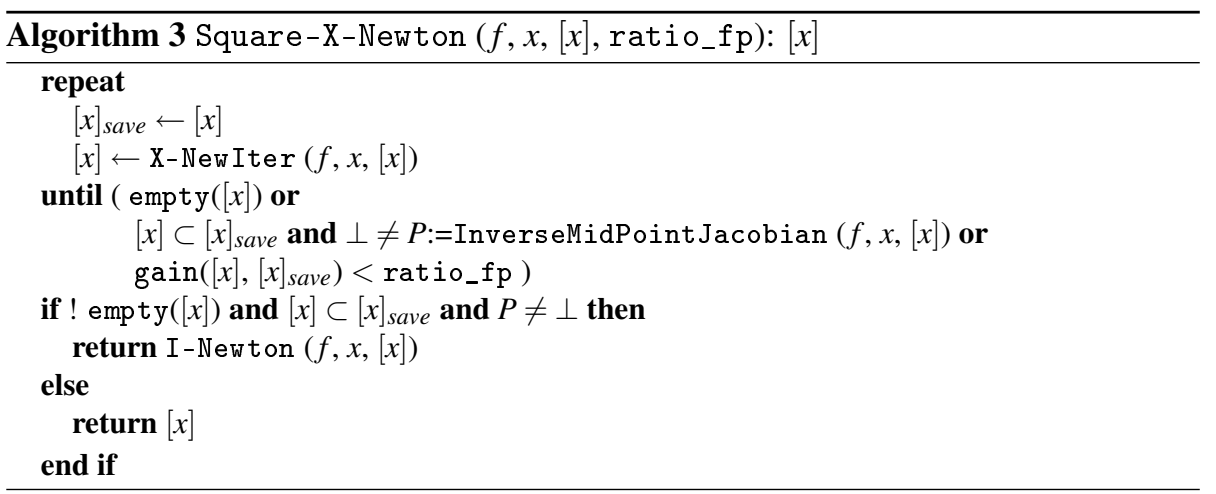

The inclusion test $[x] \subset[x]_{\text {save }}$ is a necessary condition for the existence and unicity of a solution inside $[x]{ }^{13}$ A second condition makes the test sufficient: the condition that the midpoint of the Jacobian matrix $[A]$ be invertible. This implies a so-called strong regularity condition on $[A]$ that implies its regularity [7, 24].

Therefore, in practice, each time the inclusion test is true, the function InverseMidPoint Jacobian resorts to the first two steps shown in Section A.1 for computing the preconditioning matrix $P=m([A])^{-1}$. It returns $P=\perp$ when $m([A])$ is not invertible.

Both conditions prove the existence and unicity of a solution in the box. They also imply quadratic convergence onto the linear solution set [7, 24]. Hence the last call to I-Newton.

\section{B Existence test for systems of inequality constraints}

We consider here a constraint system $S$ made of a set of inequalities, in which Algorithm 4 tries to guarantee the existence of a (floating-point) solution. Note that this existence test may fail although one such solution exists in the box, like every other existence test.

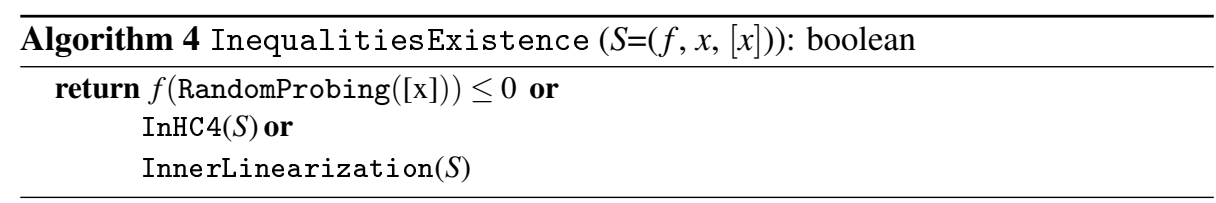

The test first randomly picks an n-dimensional point $x$ inside the box $[x]$ (see RandomProbing in Algorithm 4). If this floating-point number satisfies the constraints, i.e., $f(x) \leq 0$, then the existence test succeeds. This sometimes works in practice at the end of the combinatorial search because bisection and contraction operations have reduced the box $[x]$ around solutions. Otherwise, the test continues with more original tests based on inner boxes and inner polyhedral regions.

Definition 2 Consider a system made of only inequality constraints $f(x) \leq 0$, studied in a box $[x]^{\text {out }}$. An inner region $r^{\text {in }}$ is a feasible subset of $[x]^{\text {out }}$, i.e., $r^{\text {in }} \subset[x]^{\text {out }}$ and all points $x \in r^{\text {in }}$ satisfy $f(x) \leq 0$. An inner box $[x]^{\text {in }}$ is an inner region which is a box.

\footnotetext{
${ }^{13}$ The strict inclusion must hold in our case because the domain/bound constraints imposed by $[x]$ (i.e., $\left.x_{i} \leq x_{i} \leq \overline{x_{i}}\right)$ are yielded to the Simplex algorithm via the procedure $\mathrm{X}$-New Iter.
} 
Without detailing, InHC4 and InnerLinearization are recent heuristical algorithms able to sometimes extract respectively an inner box and an inner polytope inside a given box [8, 31]. Note that InnerLinearization uses a dual extremal interval Taylor form to extract an inner polytope [31]. In case of success of one of both inner region extraction algorithms, the existence test succeeds.

\section{B.1 Adaptation to equality constraints}

This existence test could also hold for "thick" equations, i.e., equations with a non zero-dimensional set of solutions and for relaxed equations.

A thick equation is common in practice when at least one coefficient of the equation is known with a bounded uncertainty, e.g., an imprecision on a measured distance. This also appears in equations with irrational constants, like $\pi$. Provided that the bounded uncertainties and the irrational constants are encoded by interval constants, these thick equations $f_{j}(x)=0$ are transformed, without loss of information, into two inequalities $0 \leq f_{j}(x) \leq 0$.

A "true" equality $f_{k}(x)=0$ can also be handled with a relaxation as a thick equation $f_{k}(x) \in\left[-\varepsilon_{e q},+\varepsilon_{e q}\right]$, i.e, two inequalities $-\varepsilon_{e q} \leq f_{k}(x)$ and $f_{k}(x) \leq \varepsilon_{e q}$. This is inspired by our interval $\mathrm{B} \& \mathrm{~B}$ where the default precision value $\varepsilon_{e q}=1$.e-8 for the equalities is tiny. In this case, our existence test holds for the relaxed system but not for the original one. To reach reliability for the original system, non square Newton operators, proposed by the interval analysis community (see e.g. [11]), should be applied in the end to check the existence of a real-valued solution of the system inside a given box. In practice, such a non square Newton algorithm would work with a box enclosing the inner point, box or polytope returned by Algorithm 4 . For instance, starting from the inner box returned by InHC4, the size of the considered box would be increased (e.g., doubled) on all its dimensions until the existence of a solution is guaranteed inside it or until a maximum number of iterations is reached. 


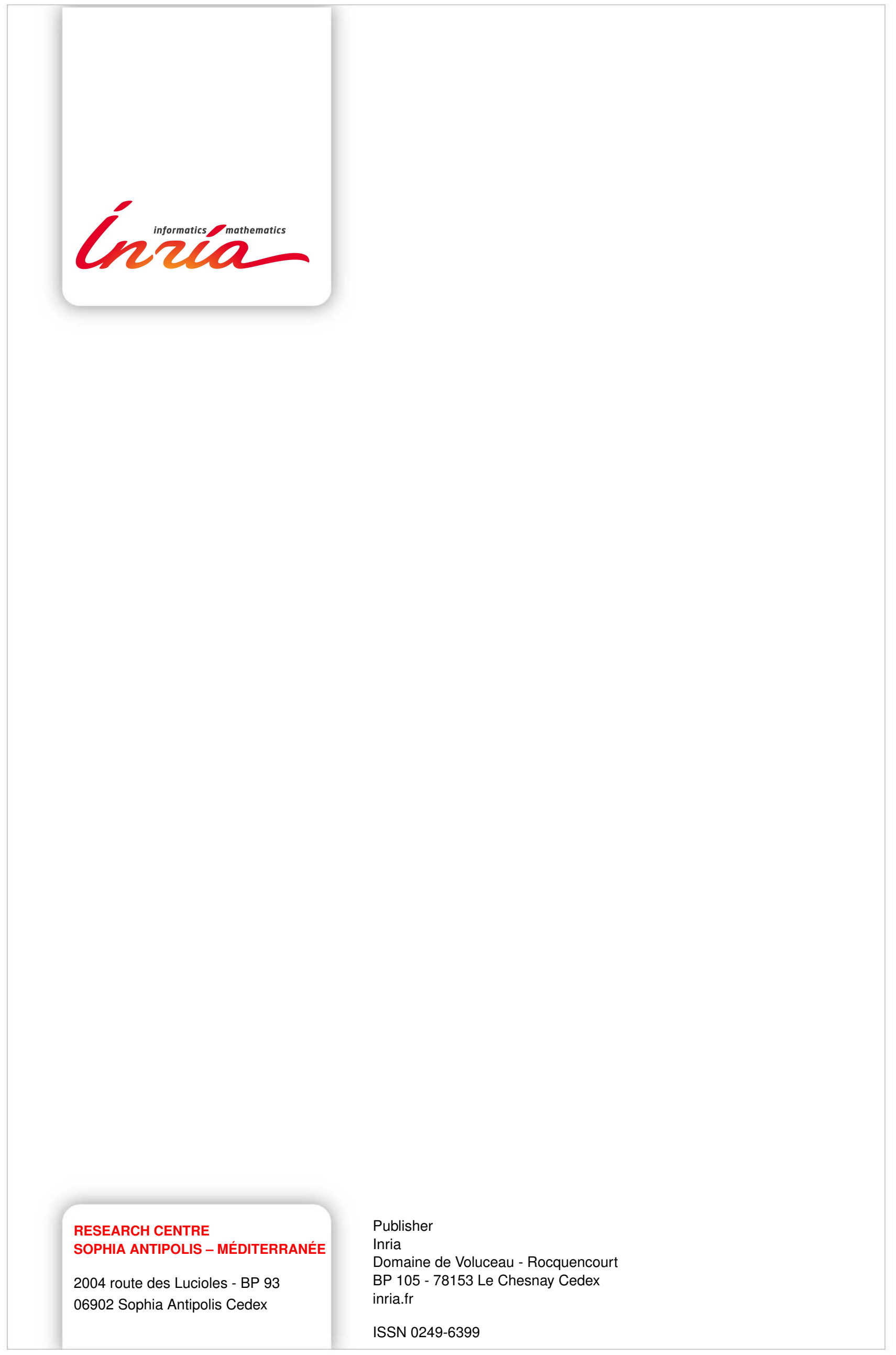

